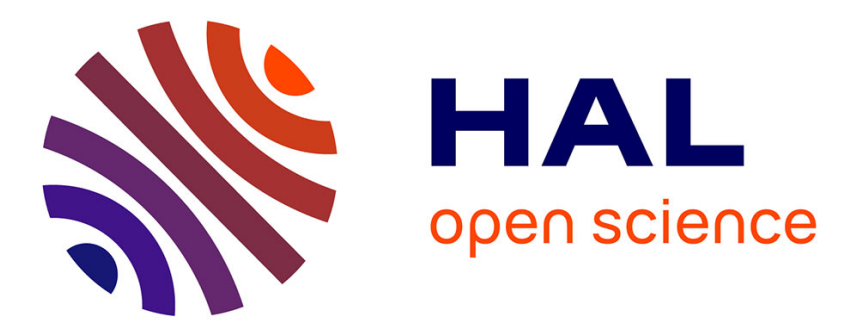

\title{
Terahertz plasmon-induced transparency and absorption in compact graphene-based coupled nanoribbons
}

\author{
Adnane Noual, Madiha Amrani, El Houssaine El Boudouti, Yan Pennec, \\ Bahram Djafari-Rouhani
}

\section{To cite this version:}

Adnane Noual, Madiha Amrani, El Houssaine El Boudouti, Yan Pennec, Bahram Djafari-Rouhani. Terahertz plasmon-induced transparency and absorption in compact graphene-based coupled nanoribbons. Applied physics. A, Materials science \& processing, 2019, 125 (3), pp.184. 10.1007/s00339019-2474-3 . hal-03143602

\section{HAL Id: hal-03143602 \\ https://hal.science/hal-03143602}

Submitted on 9 Aug 2021

HAL is a multi-disciplinary open access archive for the deposit and dissemination of scientific research documents, whether they are published or not. The documents may come from teaching and research institutions in France or abroad, or from public or private research centers.
L'archive ouverte pluridisciplinaire HAL, est destinée au dépôt et à la diffusion de documents scientifiques de niveau recherche, publiés ou non, émanant des établissements d'enseignement et de recherche français ou étrangers, des laboratoires publics ou privés. 


\title{
Terahertz plasmon-induced transparency and absorption in compact graphene-based coupled nanoribbons
}

\author{
Adnane Noual $^{1,2}$ (D) Madiha Amrani $^{1} \cdot$ El Houssaine El Boudouti $^{1} \cdot$ Yan Pennec $^{3} \cdot$ Bahram Djafari-Rouhani $^{3}$
}

\begin{abstract}
We investigate theoretically and numerically the possibility of realizing plasmon-induced transparency (PIT) and plasmoninduced absorption (PIA) in a novel compact graphene-based nanostructure. The main graphene bus waveguide is coupled to two graphene nanoribbons (GNRs). The PIT effect is obtained by setting the two GNRs in an inverted L-shape aside of the main waveguide, giving rise to lambda-like configuration in analogy with three atomic-level systems. The possibility of improving the quality factors of PIT-like resonances is shown and the associated slow light effects are showcased. The mechanism behind the observed transparency windows is related to mode splitting also known as Autler-Townes splitting phenomenon. Two PIA resonances are also demonstrated by the same system. This is achieved by inserting the two GNRs, forming an inverted T-shape, inside the main waveguide. Here the two GNRs are also set in a lambda-like configuration. We indicate the possibility of improving the Q-factor of the PIA resonances and showcase their fast light features. The PIA absorption bands are shown to be essentially caused by interference phenomena between three states as in electromagneticinduced transparency. The proposed system may help the design of tunable integrated optical devices such as sensors, filters or high speed switches.
\end{abstract}

\section{Introduction}

Recently, a great deal of attention has been paid to mimic the quantum phenomena in classical systems [1,2]. These classical analogs of quantum phenomena enable to avoid extreme required experimental conditions, and consequently, make it really easier to achieve practical applications. One of these quantum phenomena is electromagnetically induced transparency (EIT), which is a quantum effect occurring in atomic systems and caused by destructive interference between different excitation pathways to atomic levels [3]. The effects of EIT are light absorption reduction by the atomic medium and a steep dispersion over a narrow spectral region. Intense research efforts have been conducted

Adnane Noual

noualad@yahoo.fr

1 Département de Physique, Faculté des Sciences, LPMR, Université Mohamed Premier, 60000 Oujda, Morocco

2 Faculté Polydisciplinaire Nador, Université Mohamed Premier, Oujda, Morocco

3 Département de Physique, Institut d'Electronique, de Microélectronique et de Nanotechnologie, UMR CNRS 8520, Université de Lille, 59655 Villeneuve d'Ascq, France to realize the classical analog of EIT in both metal-insulator-metal (MIM) waveguide-based structures and metamaterials [4-8]. The plasmonic analog of EIT is called plasmoninduced transparency (PIT). Recently, the coupling of MIM waveguides with plasmonic resonators in the shape of nanodisks has been shown to support PIT with multiple induced resonance peaks [9-11]. PIT devices based on either MIMwaveguide structures or metamaterials are built from metallic materials. Nevertheless, it is quite hard to adjust the noble metal material permittivity, which renders their utilization in some plasmonic components rather limited. Graphene is a carbon atom thick layer with outstanding and unique optical and physical properties. Surface plasmon excitation on a graphene layer has been shown [12], and as compared to surface plasmon propagation in metals, graphene plasmons manifest a higher confinement and a relatively low propagation loss [13]. The main advantage of graphene over noble metals is the possibility to dynamically tune its conductivity by changing its Fermi-level energy; this is performed in practice by applying a voltage gate on graphene layer [14]. Some years ago, the utilization of graphene and plasmonics has sparked a large body of research to design graphene-based metasurfaces [15, 16], photo-detectors [17, 18], optical waveguides [19, 20], refractive index sensors 
[21, 22] and perfect multi-band plasmonic absorbers [23]. Furthermore, tunable PIT resonances have been realized in graphene-based waveguide structures [24], and important PIT effects were recently achieved in two-layer crossed graphene nanoribbon-based nanostructure [25].

In addition, plasmon-induced absorption (PIA) is a similar phenomenon to PIT giving rise to a sharp dip in the transmission spectrum of the system and an enhancing absorption. PIA arises due to extreme destructive interferences between dark and bright modes [26]. Recently, PIA has been investigated in metamaterial-based nanostructures $[27,28]$. It has been shown that PIA can play a significant role in the realization of $\mathrm{THz}$-integrated spectral control and the design of ultra-fast optical switching [29], information storage [30] and optical buffers [31]. However, similar to electromagnetic-induced transparency (EIT), a plasmon transparency window may be observed only due to the interaction between modes that causes their splitting, a phenomenon known as Autler-Townes splitting (ATS) [32-34]; it consists in field-driven splitting of the optical response of the system. In particular, unlike EIT-effect no Fano interferences are involved in ATS phenomenon.

In this paper, we investigate theoretically, based on coupled mode theory (CMT), and numerically, based on finite element method (FEM), PIT-like effect and PIA phenomenon in a compact graphene-based waveguide nanostructure. The geometry design of the proposed device consists in a graphene bus waveguide (GBW) and two graphene nanoribbons (GNRs) set in an inverted L-shape or T-shape, giving rise to a lambda-like state in analogy with three atomic-level system. The two GNRs are, respectively, inserted inside or aside of the main waveguide as depicted in Fig. 1a, b. These proposed designs enable the control of the Q-factor of the PIT(-like)/PIA resonances by adjusting the appropriate parameters of the structure. Moreover, to the best of our knowledge, these designs aimed at realizing PIT(-like)/ PIA resonances have not been reported before in the literature. We demonstrate that PIT(-like) resonances are mainly caused by mode splitting or ATS-effect, whilst the PIA dips are shown to arise fundamentally because of EIT phenomenon. The graphene waveguide and the two GNRs should be both embedded in a dielectric material such as $\mathrm{SiO}_{2}$, to fit practical applications [35].

In that spirit, the proposed device is placed in a dielectric medium with a dielectric permittivity $\varepsilon=2.25$.

\section{Model and simulation method}

The in-plane cross section ( $x y$-plane) of the structure is investigated and analyzed based on FEM method. Specifically, we use COMSOL Multiphysics to perform the numerical simulations. In our model, we treat graphene as a very
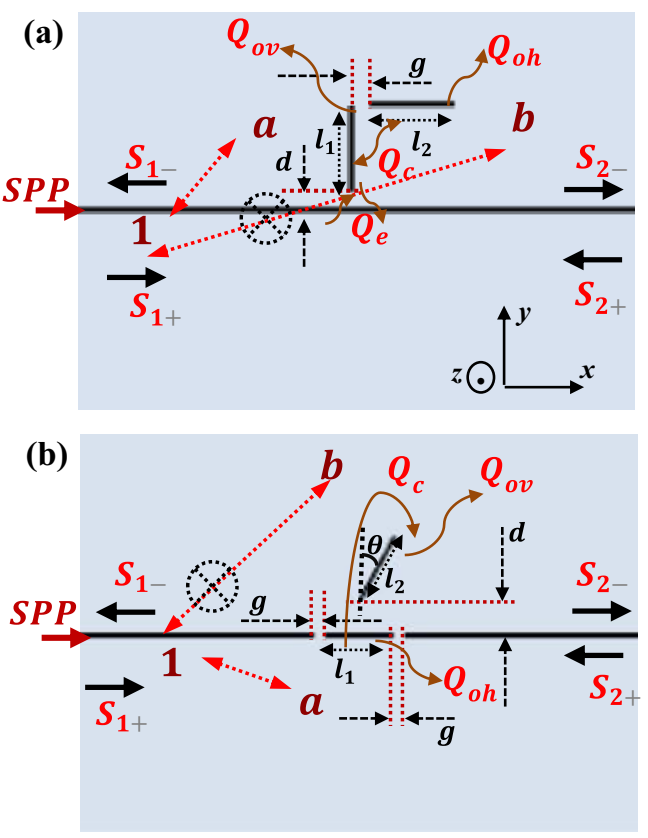

Fig. 1 a Geometry of a graphene-based waveguide structure sidecoupled to two graphene nanoribbons forming an inverted L-shape letter shape. b Same as in a but the two ribbons are inserted inside the waveguide, and set in an inverted T-shape. The three atomic levels represented as $\mid 1>$, $\mid \mathrm{a}>$ and $\mid \mathrm{b}>$ (in analogy with atomic systems) are sketched within the structure in both configurations (a) and (b)

thin anisotropic material with a thickness $\Delta$, which we chose throughout the paper to be equal to $1 \mathrm{~nm}$. The effective surface permittivity of graphene is given by $\varepsilon_{\mathrm{s}}=1+\sigma / \varepsilon_{0} \omega \Delta$ [36], while its normal permittivity is a simple constant $\varepsilon_{\perp}=2.5$. Here, $\sigma$ is the surface conductivity of the graphene, $\varepsilon_{0}$ is the vacuum permittivity and $\omega$ is the angular frequency. In the terahertz frequency domain, $\sigma$ takes the form of a Drude-like expression, $\sigma=i e^{2} E_{\mathrm{F}} / \pi \hbar^{2}\left(\omega+i \tau^{-1}\right)$ [37], where $e, E_{\mathrm{F}}, \hbar, \tau$ are the electron charge, the Fermi energy level, the reduced Planck constant and the relaxation time, respectively. The carrier relaxation time is related to the Fermi velocity, $v_{\mathrm{F}}=10^{6} \mathrm{~m} / \mathrm{s}$ [24], and to the carrier mobility $\mu$ as $\tau=\mu E_{\mathrm{F}} / e v_{\mathrm{F}}^{2}$. We assumed a value of $10^{5} \mathrm{~cm}^{2} \mathrm{~V}^{-1} \mathrm{~s}^{-1}$ for $\mu$ as in [24]. The Fermi energy level of the two GNRs is identical and is referred to as $E_{\mathrm{F} 1}$, whereas $E_{\mathrm{F} 0}$ corresponds to the GBW Fermi energy level. A fixed value for $E_{\mathrm{F} 0}$ is set throughout this paper such as $E_{\mathrm{F} 0}=140 \mathrm{meV}$. To get rid of any possible reflections perturbing the system, we put up PML layers on the bottom and upper sides of the structure. Numerical port boundary condition is applied at the input (left side) and output (right side) sides of the system. Extremely fine triangular mesh is set within PML layers, whereas a finer (triangular) mesh is used in the dielectric material. This ensures to have more than five points per wavelength in these domains. In the graphene, we employ a 
triangular mesh with maximum element size such as $1 \mathrm{~nm} \times 0.2 \mathrm{~nm}$, that is, five elements along the graphene thickness. Numerical tests show that the results do not evolve if finer mesh schemes are applied, which indicate that the implemented mesh is sufficient for the numerical convergence of the results. A TM-polarized SPP-eigen mode of the GBW is excited and launched from the left port; the transmission spectrum is then recorded at the right end of the waveguide. In this paper, the transmission coefficient is defined as the ratio between the output powers measured at the right end port with and without the two GNRs.

\section{Plasmon-induced transparency}

In this section, PIT-like resonances are shown in the geometry design sketched in Fig. 1a with the L-shape GNRs. The parameters of the system depicted in this figure are set as follows: $d=60 \mathrm{~nm}, g=10 \mathrm{~nm}, l_{1}=l_{2}=250 \mathrm{~nm}$ and $E_{\mathrm{F} 1}=160 \mathrm{meV}$. In Fig. 2a, we present the corresponding transmission spectrum recorded at the right end port of the structure (black curve). For comparison purposes, we also give in Fig. 2a the transmission spectrum when a single ribbon horizontally (red curve) or vertically (blue curve) set is side-coupled to the GBW. In the case of one vertical ribbon, two SPP-eigen modes are found in the frequency range of interest [4.75, 18.25] THz. One can note that the high frequency mode is not excited when the ribbon is horizontally set. This is related to the distance of the ribbon to the waveguide which is quite higher in this case and to the high confinement of this mode around the ribbon. Indeed, the low frequency mode is excited (although with less intensity as compared to the vertical ribbon); and its magnetic field map (not shown here) indicates that the second order mode is strongly localized around the ribbon as opposed to the first order mode. Hence, at such far distances, the mode in the GNR does not couple well with the guided mode and is not excited. Now, when both ribbons are coupled to the GBW (black curve in Fig. 1a), we notice the emergence of two resonance peaks around $10.5 \mathrm{THz}$ (low frequency peak) and $16.2 \mathrm{THz}$ (high frequency peak) separated by a transparency window. Each transmission window is surrounded by two transmission dips. The transmission peaks are plasmon-like induced transparency (PIT-like) resonances of the system. The comparison of the transmission of the system with the transmission spectra of the identical single ribbons is taken alone (Fig. 2a), indicate that the coupling of the latter causes the splitting of their transmission dips, giving rise to four dips either symmetric or anti-symmetric as shown in Fig. 2b, $\mathrm{d}, \mathrm{e}, \mathrm{g}$ ) by the $z$-component of their magnetic field maps. The symmetry is defined with respect to the symmetry plane $C_{y x}$ of the ribbons, which is highlighted by white dashed curve in the figures. In particular, the $H_{\mathrm{z}}$-field maps at the PIT-like

(a)

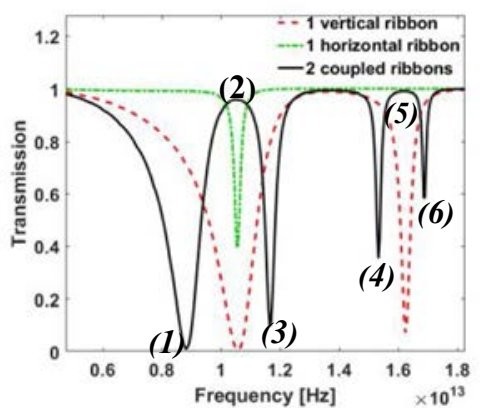

(b)

(c)

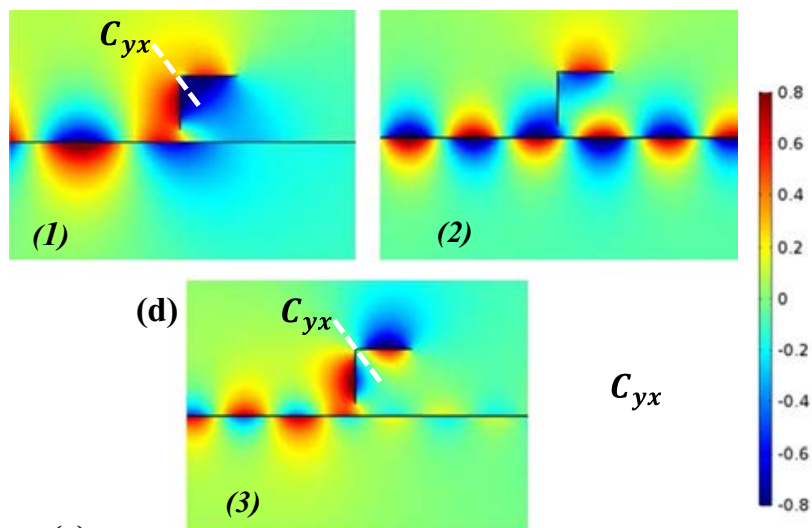

(e)

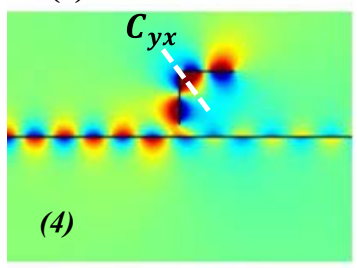

(g)

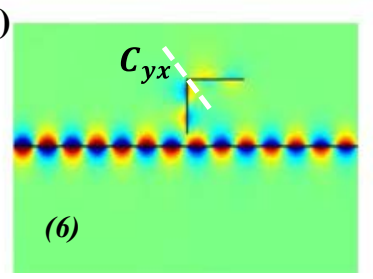

Fig. 2 a Transmission spectra of the structure depicted in Fig. 1a for $d=60 \mathrm{~nm}, g=10 \mathrm{~nm}, l_{1}=l_{2}=250 \mathrm{~nm}$ and $E_{\mathrm{F} 1}=160 \mathrm{meV}$. Transmission spectra when a single ribbon is either vertically or horizontally side-coupled to the waveguide are also shown. b-g $H_{z}$-field map within the structure at the transmission dips and peaks referred to as (1)-(6) in (a), respectively. Notice the different color scales between $\mathrm{b}-\mathrm{d}$ and $\mathrm{e}-\mathrm{g}$. Symmetry plane $C_{y x}$ of the two ribbons is highlighted in dashed white curve

resonances (Fig. 2c, f) reflect a strong coupling between the ribbons, leading to the suppression of the bright mode (lower vertical ribbon). The physical mechanism behind the observed resonance peaks will be discussed in more details later on in this section.

In the light of temporal coupled mode theory (CMT), the transmission properties of the system could be fairly well 
captured and fitted. Indeed, if we denote by $a_{\mathrm{v}}$ and $a_{\mathrm{h}}$ the energy mode amplitudes of the lower vertical ribbon (bright mode) and the upper horizontal ribbon (dark mode), respectively, then we can write [38, 39]:

$\frac{\mathrm{d} a_{\mathrm{v}}}{\mathrm{d} t}=\left(-j \omega_{\mathrm{v}}-\frac{\omega_{\mathrm{v}}}{2 Q_{\mathrm{ov}}}-\frac{\omega_{\mathrm{v}}}{2 Q_{\mathrm{e}}}\right) a_{\mathrm{v}}+j \sqrt{\frac{\omega_{\mathrm{v}}}{2 Q_{\mathrm{e}}}}\left(S_{1+}+S_{2+}\right)+j \frac{\omega_{\mathrm{h}}}{2 Q_{\mathrm{c}}} a_{\mathrm{h}}$,

$\frac{\mathrm{d} a_{\mathrm{h}}}{\mathrm{d} t}=\left(-j \omega_{\mathrm{h}}-\frac{\omega_{\mathrm{h}}}{2 Q_{\mathrm{oh}}}\right) a_{\mathrm{h}}+j \frac{\omega_{\mathrm{v}}}{2 Q_{\mathrm{c}}} a_{\mathrm{v}}$,

where $\omega_{\mathrm{v}}$ and $\omega_{\mathrm{h}}$ are the eigenfrequencies of the vertical and the horizontal ribbons, respectively. $Q_{\mathrm{ov}}, Q_{\mathrm{oh}}, Q_{\mathrm{e}}$ and $Q_{\mathrm{c}}$ are the quality factors related to intrinsic losses, waveguide coupling loss and coupling strength between the two GNRs, respectively. On the other hand, $S_{i+}$ and $S_{i-}$ are, respectively, the amplitude of the input and output SPP waves, where the subscript $i=1,2$ represents the left and right ports (resp.) of the GBW. It should be noted that $\left|S_{i \pm}\right|^{2}$ represents the power carried out by the SPP waves. In our model, we set $S_{2+}=0$, that is, no SPP wave is launched from the right port. Based on time reversal symmetry and energy conservation, one gets the following relationships between the incoming and outgoing SPP waves:

$S_{2-}=S_{1+}-j \sqrt{\frac{\omega_{\mathrm{v}}}{2 Q_{\mathrm{e}}}} a_{\mathrm{v}}$,

$S_{1-}=S_{2+}-j \sqrt{\frac{\omega_{\mathrm{v}}}{2 Q_{\mathrm{e}}}} a_{\mathrm{v}}$.

The combination of Eqs. (1)-(4) leads to the transmission spectrum of the structure:

$T=\left|\frac{S_{2-}}{S_{1+}}\right|^{2}=\left|1-\frac{1}{Q_{\mathrm{e}}} \cdot \frac{j 2 \delta_{\mathrm{h}}+\frac{1}{Q_{\mathrm{oh}}}}{\left(j 2 \delta_{\mathrm{v}}+\frac{1}{Q_{\mathrm{ov}}}+\frac{1}{Q_{\mathrm{c}}}\right)\left(j 2 \delta_{\mathrm{h}}+\frac{1}{Q_{\mathrm{oh}}}\right)+\left(\frac{1}{Q_{\mathrm{c}}}\right)^{2}}\right|^{2}$,

where $\delta_{\mathrm{h}, \mathrm{v}}=\left(\omega-\omega_{\mathrm{v}, \mathrm{h}}\right) / \omega_{\mathrm{v}, \mathrm{h}}$ is used so to normalize the incident frequency. In our model, we choose $\delta_{\mathrm{h}}=\delta_{\mathrm{v}}$ (that is, $\left.\omega_{\mathrm{v}}=\omega_{\mathrm{h}}\right)$. Let us mention that the intrinsic quality factors $Q_{\mathrm{oi}}$ $(i=h, v)$ can be estimated using $Q_{\mathrm{oi}}=\operatorname{real}\left(n_{\mathrm{effi}}\right) / 2 \operatorname{Im}\left(n_{\mathrm{effi}}\right)$ [38], where $n_{\text {effi }}=k_{\text {sppi }} / k_{0}$ is the effective refractive index of the SPP wave propagating along the GNR $i, k_{\text {sppi }}$ is the SPP propagation constant and $k_{0}$ is the wave vector in vacuum. On the other hand, $Q_{\mathrm{e}}$ and $Q_{\mathrm{c}}$ are evaluated as fitting parameters. Given that in the frequency range of interest the system exhibits two PIT-like resonances, we shall follow the approach of [40] to get the total transmission spectrum. One gets

$T=T_{1} \times T_{2}$,
$T_{i},(i=1,2)$ being the transmission coefficient of the system associated with the resonance frequency $\omega_{i}$. In Fig. 3, we give the transmission spectrum provided by the CMT model along with the FEM-based numerical results. The parameters of the structure are chosen as in Fig. 2a. One notes that a quite good agreement is obtained between theoretical results based on CMT model and numerical simulations, despite some small discrepancies (in terms of transmission values). We attribute these to the rough approximations of the intrinsic quality factors $Q_{\mathrm{oi}}(i=h, v)$ of the GNRs and the approximation of the CMT model. Nevertheless, both theoretical and numerical curves produce a quasi-identical profile, so that the CMT-based fitting can significantly help understanding the numerical transmission spectra. It is worth mentioning that the fitted parameters for the low frequency resonance are found as $Q_{\mathrm{e}} \approx 7.8586, Q_{\mathrm{c}} \approx 7.7425$ and $\omega_{\mathrm{v}}=\omega_{\mathrm{h}} \approx 10.365 \mathrm{THz}$. For the high frequency resonance, one gets $Q_{\mathrm{e}} \approx 125.88$, $Q_{\mathrm{c}} \approx 9.87$ and $\omega_{\mathrm{v}}=\omega_{\mathrm{h}} \approx 16.18 \mathrm{THz}$.

To figure out if the observed PIT-like resonances in the transmission spectrum of the system are originated by EIT or Autler-Townes splitting (ATS) [32-34], we proceeded to the fitting of the numerical results based on the equations provided in [32-34] where the difference between both phenomena is highlighted. In the case when the transparency window is caused by ATS, the numerical spectrum is fitted by two Lorentzian lines having the same sign so as to signify the absence of Fano interferences. The transmission coefficient reads in this case:

$T_{\mathrm{ATS}}=1-\frac{C_{1}}{\left(\omega-\delta_{1}\right)^{2}+\Gamma_{1}^{2}}-\frac{C_{2}}{\left(\omega-\delta_{2}\right)^{2}+\Gamma_{2}^{2}}$,

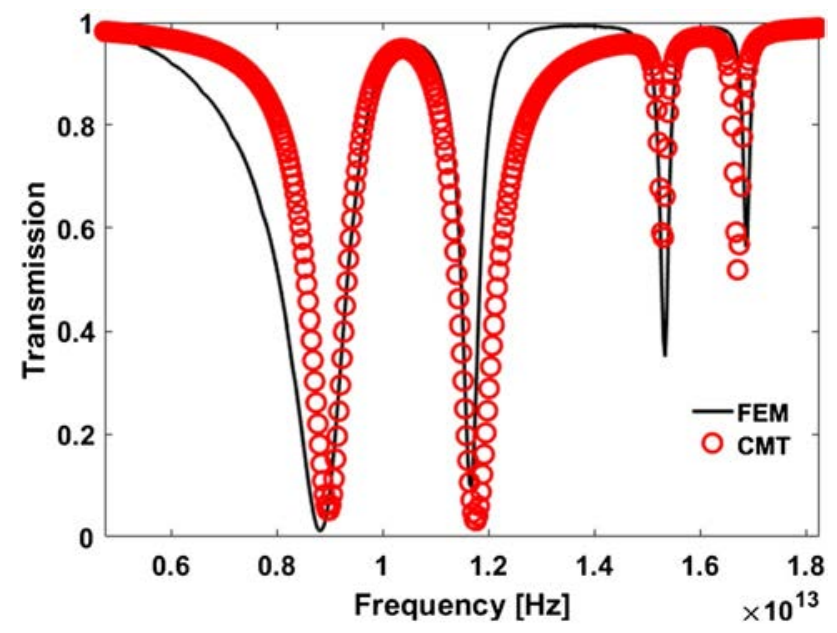

Fig. 3 Transmission spectra provided by the CMT model (red circles) and the FEM method (black solid line). The parameters of the structure are chosen as in Fig. 2a 
where $C_{k}, \delta_{k}$ and $\Gamma_{k}(k=1,2)$ are the free fitting parameters which can be estimated from the numerical transmission spectrum.

On the other hand, the EIT-fitting function is such that the two Lorentzians have opposite signs, thereby reflecting the presence of Fano interferences. One has

$T_{\mathrm{EIT}}=1-\frac{C_{1}}{\left(\omega-\omega_{\mathrm{R}}\right)^{2}+\Gamma_{1}^{2}}+\frac{C_{2}}{\left(\omega-\omega_{\mathrm{R}}\right)^{2}+\Gamma_{2}{ }^{2}}$.

Similarly, $C_{k}$ and $\Gamma_{\mathrm{k}}(k=1,2)$ are the free fitting parameters whilst $\omega_{\mathrm{R}}$ is the position of the PIT-like resonance peak. In Fig. 4a, b, we present the transmission spectra based on ATS-fitting function together with the FEM spectrum around the low and high frequency resonances, respectively. The structure parameters are set as in Fig. 2a. We have checked that the EIT-function provides a quite poor fitting of the FEM results (not shown here) around both the low and high
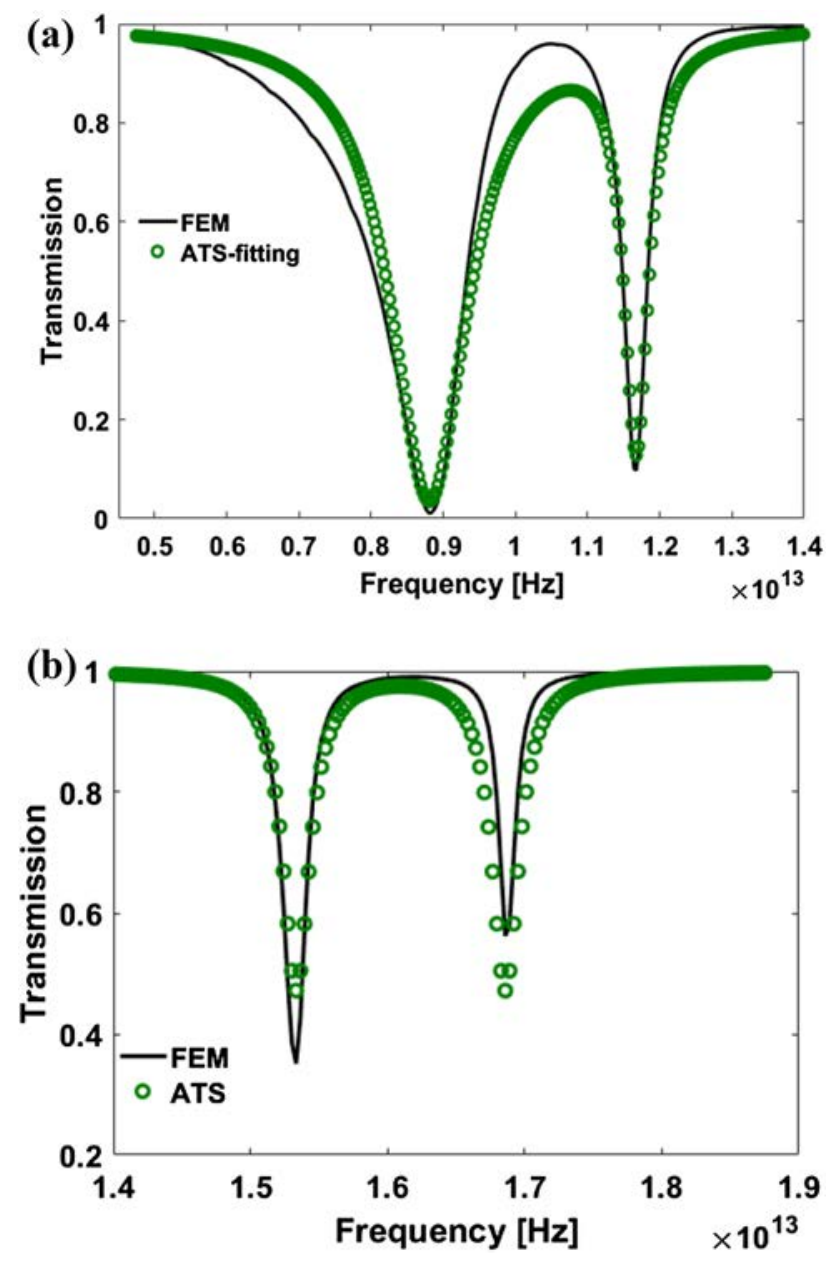

Fig. 4 a Transmission spectra based on FEM method (black solid line) and ATS-fitting results (green curve) around the low frequency resonance. $\mathbf{b}$ Same as in a but around the high frequency resonance frequency resonances. Conversely, it can be seen in Fig. 4a that the ATS fitting is very close to the simulation result at the low frequency resonance. The same trend is observed around the high frequency mode (Fig. 4b), where the ATS function provides an almost perfect fitting. Hence, both PITlike peaks are mainly caused by ATS effect rather than EIT phenomenon. As explained in [32-34], these results indicate that the lower vertical GNR (bright-mode) is strongly coupled to the upper horizontal ribbon (dark-mode). In other words, the two GNRs lie in the strong coupling regime in which the mode splitting is mainly attributed to ATS. To dig deeper in this, we have progressively increased the coupling parameter monitoring the coupling intensity between the two GNRs, that is, the metallic gap $g$ (Fig. 1a). The corresponding results are depicted in Fig. 5a.

We observe that the low frequency PIT-like peak gets more and more narrow and that the full width at half maximum (FWHM) of the two dips surrounding it decreases down asymmetrically as $g$ increases. The behavior of the high frequency resonance is similar in that the resonance peak gets narrower while $g$ is increased. Nevertheless, we notice a small difference, which is that the FWHM of the dips surrounding the resonance peak gets decreased down symmetrically with $g$. This can be explained by referring to the $\mathrm{z}$-magnetic field maps depicted in Fig. 2b, $\mathrm{g}$ on the one hand.

Indeed, the first supermode (Fig. 2b) seems more intensely coupled to the GBW as compared to the second supermode in Fig. 2d, which is more localized within the GNR. On the other hand, the supermodes depicted in Fig. 2e, g reflect a rather equivalent coupling strength with the GBW as both these modes are more localized around the GNRs and less coupled to the GBW.

In the following, we shall check if in the range of the high values of $g$ (Fig. 5a), the PIT-like resonances shall be fitted by EIT or ATS corresponding functions. To that end, we have performed the EIT/ATS fitting of the numerical results for $g=80 \mathrm{~nm}$, while keeping the remaining parameters as before. The results are depicted in Fig. 5b (low frequency resonance) and 5c (high frequency resonance). One notices that the EIT function provides a much better fitting as compared to the case when $g=10 \mathrm{~nm}$, especially around the high frequency resonance. Nevertheless, the ATS-fitting function still approaches better the numerical results in comparison with the fitting provided by the EITbased function. This is particularly true around the high frequency mode where the ATS-fitting is almost perfect. Overall, the observed trends indicate that at $g=80 \mathrm{~nm}$ we are actually in an intermediate situation where the resonances fit more or less both ATS and EIT phenomena [32] even though ATS is a preponderant mechanism as compared to EIT phenomenon. 

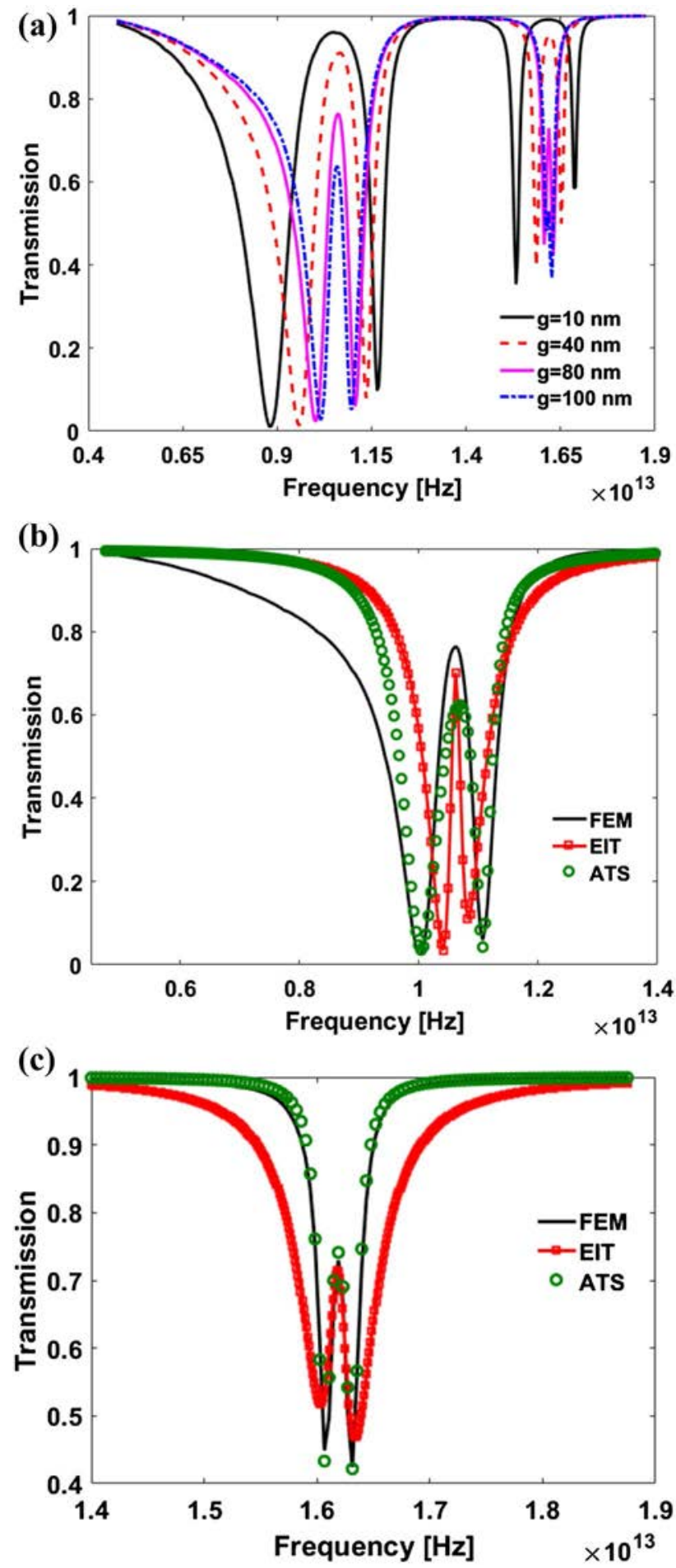

Fig. 5 a Transmission spectra of the structure in Fig. 1a for various values of $g$. The remaining parameters are fixed as in Fig. 2a. b ATS/EIT-fitting results of the FEM spectrum at the low frequency resonance for $g=80 \mathrm{~nm}$. $\mathbf{c}$ Same as in $\mathbf{b}$ but at around the high frequency resonance

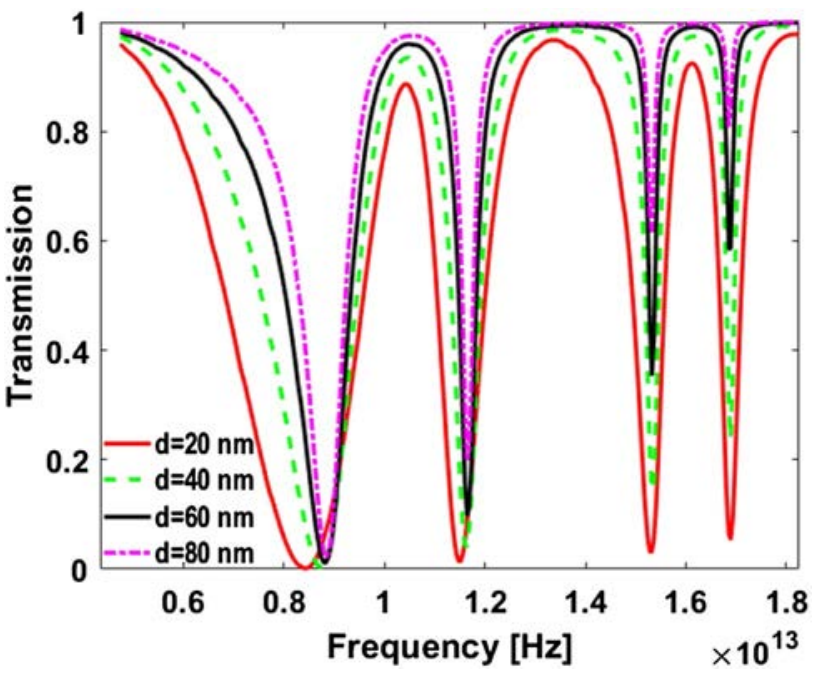

Fig. 6 Transmission spectra of the system (of Fig. 1a) for different values of $d$, namely $d=20,40,60$ and $80 \mathrm{~nm}$. The rest of the parameters are kept constant as in Fig. 2a

The results of Fig. 5a demonstrate the possibility of improving the Q-factors of the resonance peaks by adjusting the metallic gap $g$. For instance, for $g=25 \mathrm{~nm}$, the Q-factor of the low frequency peak is about $Q_{1} \approx 7$, whereas the second order mode Q-factor is $Q_{2} \approx 19$. The increase of $g$ up to $g=80 \mathrm{~nm}$ results in improved Q-factors such as, for the low and high frequency resonances, one gets $Q_{1} \approx 17.2$ and $Q_{2} \approx 127$, respectively.

Another means by which one can further improve the $Q$ values is to adjust the parameter $d$ (Fig. 1a) representing the coupling strength between the GBW and the lower vertical GNR (bright mode). Figure 6 presents the transmission spectrum of the system for different values of $d$. The remaining parameters are set as in Fig. 2a. The evolution of the transmission coefficient of the system versus $d$ shows the improvement of the Q-factor associated with the PIT-like resonances as $d$ decreases. Indeed, increasing $d$ diminishes the coupling strength between the GBW and the vertical ribbon, which results in a narrower dip of the bright mode and also the dark mode (because of their interaction). As a consequence, the FWHM of the PIT peaks surrounded by the bright and dark mode dips increases. Hence, by appropriately tuning the parameters $d$ and $g$, one can get fairly good Q-values of the two PIT-like resonances.

The shape of the transmission spectrum for $d=20 \mathrm{~nm}$ seems quite different from the other spectra for $d>20 \mathrm{~nm}$, especially all the dips reach almost zero and the FWHM of the two first dips are quite large. This is probably due to the high interaction between the vertical ribbon and the main guide (Fig. 1a) for this value.

Alongside the study of the transmission amplitude, it is quite instructive to analyze the dispersion properties 


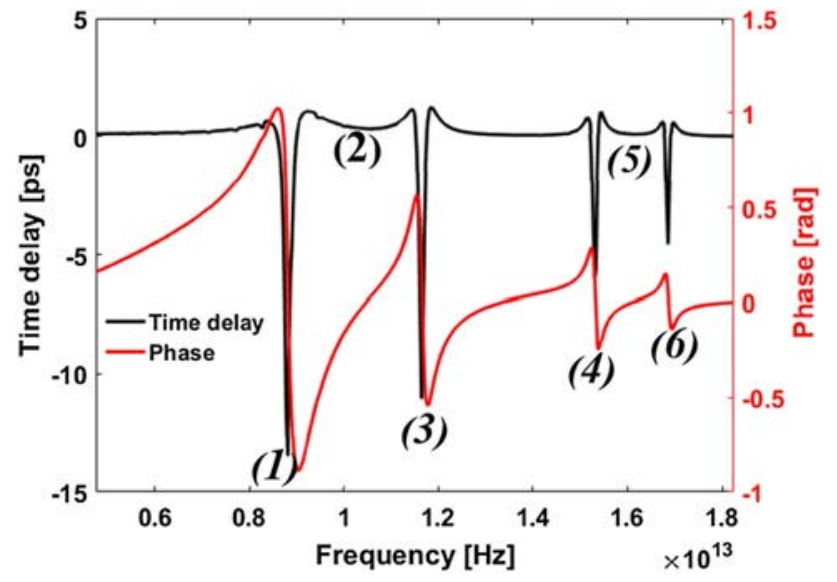

Fig. 7 Phase and delay time of the structure of Fig. 1a as a function of the frequency. The parameters of the structure are chosen as in Fig. 2a

of the proposed structure (Fig. 1a). To that end, we have plotted together in Fig. 7 the delay time and the transmission phase evolution as a function of the frequency. The parameters of the system are set such as: $d=60 \mathrm{~nm}$, $g=10 \mathrm{~nm}, l_{1}=l_{2}=250 \mathrm{~nm}$ and $E_{\mathrm{F} 1}=160 \mathrm{meV}$. The delay time is defined as $\tau=-\frac{\mathrm{d} \varphi}{\mathrm{d} \omega}$, where $\varphi$ is the phase of the transmission coefficient. It is worthwhile to recall that the delay time represents physically the time spent by the light waves captured by the two GNRs before their transmission to the output. Steep phase drops can be observed around the transmission dips labeled (1), (3), (4) and (6) in Fig. 2a, whilst around the induced transparency domains labeled (2) and (5) the phase becomes steeply positive. On the other hand, the time delay turns negative at the transmission dips and gets higher within the transparency windows, indicating the falling down of the group velocity (i.e., slow light effect).

The PIT-like resonances may be readily adjusted by simply shifting the Fermi energy levels of the ribbons. To show that, we have calculated the transmission spectra of the system for different values of $E_{\mathrm{F} 1}$. The remaining parameters are left unchanged as previously. Figure 8 presents the corresponding results where it can be seen that the resonance peaks are shifted towards high frequencies with increasing $E_{\mathrm{F} 1}$. The observed trend can be easily explained if one refers to the relation giving the resonance wavelength of a single graphene nanoribbon embedded in a dielectric; this relation reads [41]:

$\lambda=\left(\frac{2 \pi \hbar c}{e}\right) \sqrt{\frac{\eta \varepsilon_{0} \varepsilon_{\mathrm{r}} l}{E_{\mathrm{F}}}}$,

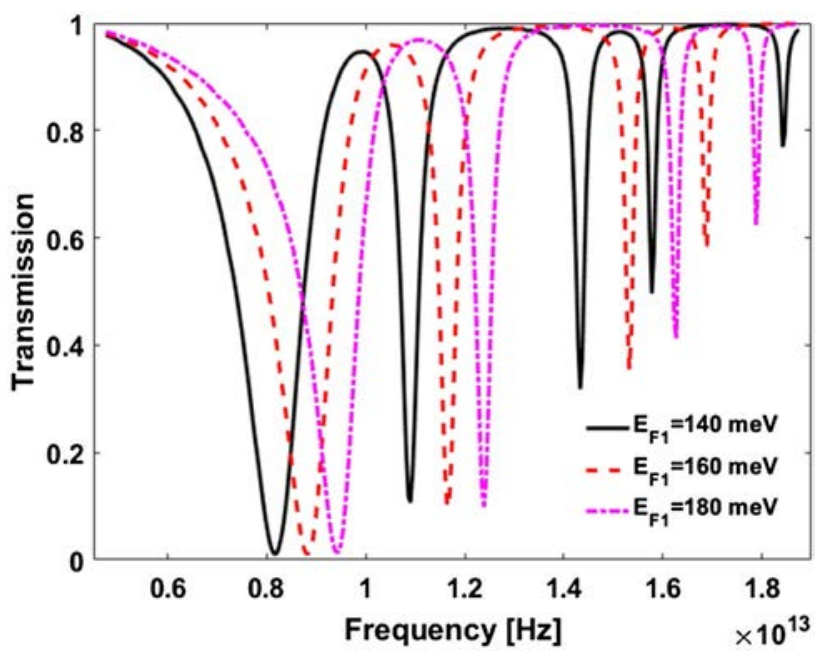

Fig. 8 Transmission spectra of the structure of Fig. 1a for different values of $E_{\mathrm{F} 1}$. The rest of the parameters are kept unchanged as in Fig. 2a

where $l$ is the nanoribbon length, and $\eta$ is a dimensionless fitting parameter (from the simulations data). Based on this equation, the resonance wavelength is proportional to the inverse of the square root of the Fermi energy level of the GNRs, which is coherent with the obtained numerical results of Fig. 8.

\section{Plasmon-induced absorption}

Now, we focus on the geometry design sketched in Fig. 1b, where the lower graphene ribbon is inserted horizontally along the GBW, whereas the upper ribbon, placed at a distance $g$ from the GBW, is tilted at an angle $\theta$ from the y-axis. In this configuration, the two ribbons couple evanescently through the near field with the two decoupled branches of the GBW, and transmits only frequencies corresponding to their eigen-SPP modes to the output. The filtered modes appear as transmission peaks in the transmission spectrum. Figure 9a gives the transmission spectrum versus frequency for each ribbon coupled alone with the GBW (black and green curves). The parameters of the system are set such as $l_{1}=l_{2}=250 \mathrm{~nm}, E_{\mathrm{F} 1}=160 \mathrm{meV}$, and $g=10 \mathrm{~nm}$ for the horizontal ribbon. For the inclined ribbon, we choose $\theta=0^{\circ}$ and $d=120 \mathrm{~nm}$. Two transmission modes of the horizontal ribbon are observed in the frequency range of interest, whilst the vertically ribbon (above the GBW) gives rise to the first order transmission mode only (with low intensity), while the second mode vanishes. This is due to the fact that the vertical ribbon is weakly coupled to the waveguide being far apart, especially for the second order mode frequency. Indeed, as mentioned above this mode does not couple well 
Fig. 9 a Transmission spectrum of the structure of Fig. 1b when a single ribbon is coupled to the waveguide with $l_{1}=250 \mathrm{~nm}$ and $g=10 \mathrm{~nm}$ for the horizontal ribbon (black curve). For the ribbon shifted above the waveguide (green curve), the parameters are: $l_{2}=250 \mathrm{~nm}, d=120 \mathrm{~nm}$ and $\theta=0^{\circ}$. The Fermi energy level of the ribbons is $E_{\mathrm{F} 1}=160 \mathrm{meV}$. b Same as in a but when both ribbons are present in the structure. $\mathbf{c}-\mathbf{h}$ z-component of the magnetic field map at the transmission dips and peaks frequencies labeled (1)-(6), in $\mathbf{b}$, respectively

with the waveguide at such far distances because of its strong confinement around the GNR. In Fig. 9b, we have calculated the transmission spectrum of the structure depicted in Fig. $1 \mathrm{~b}$ when both ribbons are present.

Two transmission dips labeled (2) and (3) occur between new emerging transmission peaks. The associated absorption coefficient (not given here) of the system shows an enhanced absorption at the transmission dips. Therefore, the induced transmission dips correspond to plasmon-inducedlike absorption resonances. The physical mechanism behind these resonances is related to the interaction of the identical ribbons, giving rise to the splitting of the transmission modes of one ribbon. The resulting new peaks are rather either symmetric or anti-symmetric with respect to the symmetry plane parallel to the vertical ribbon. This can be seen in Fig. 9c, e, $\mathrm{f}, \mathrm{h}$, where we give the $z$-component of the magnetic field map at the transmission peak frequencies.

On the other hand, the magnetic field map at the induced dips depicted in Fig. 9d-g, indicates that the upper ribbon is strongly excited, whereas the lower one is much less excited. To determine which from EIT and ATS fits well the observed PIA-like dips, we will proceed as before (see below) to the fitting of the numerical results based on EIT/ ATS-fitting functions.

Following the same theoretical approach [42] developed in the previous section, we can obtain the theoretical transmission coefficient of the structure (Fig. 1b) based on the CMT model such as

$$
\begin{aligned}
T & =\left|\frac{S_{2-}}{S_{1+}}\right|^{2} \\
& =\left|\frac{1}{Q_{e}} \cdot \frac{j 2 \delta+\frac{1}{2 Q_{o v}}}{\left(j 2 \delta+\frac{1}{4 Q_{o h}}+\frac{1}{4 Q_{o v}}\right)^{2}+\left(\frac{1}{2 Q_{c}}\right)^{2}-\left(\frac{1}{4 Q_{o h}}-\frac{1}{4 Q_{o v}}+\frac{1}{Q_{e}}\right)^{2}}\right|^{2},
\end{aligned}
$$

where $\delta=\left(\omega-\omega_{v, h}\right) / \omega_{v, h}$ and $\omega_{v}=\omega_{h}=\omega_{v, h}$ in the case where no detuning is set between the two GNRs as in this work. As before, $Q_{\mathrm{ov}}, Q_{\mathrm{oh}}, Q_{\mathrm{e}}$ and $Q_{\mathrm{c}}$ refer to the quality factors related to intrinsic loss, waveguide coupling loss and coupling strength between the two GNRs, respectively. The CMT-based fitting results along with the FEM-based numerical spectrum of the system are presented in Fig. 10a. (a)

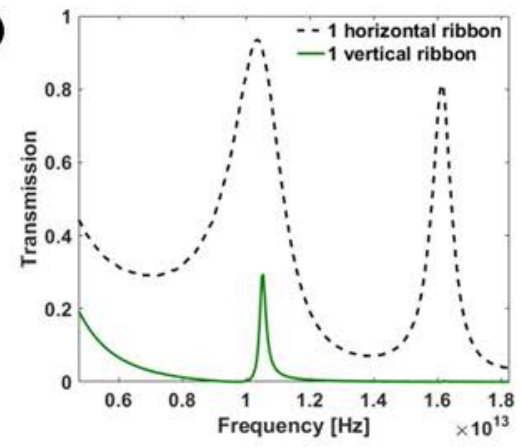

(b)
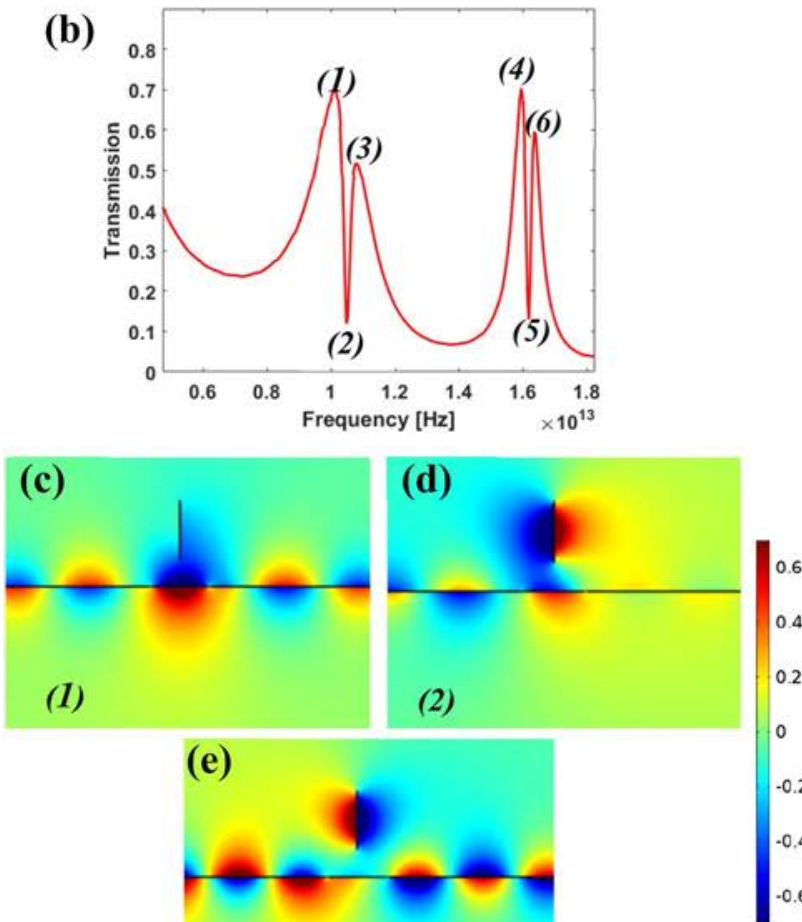

(3)
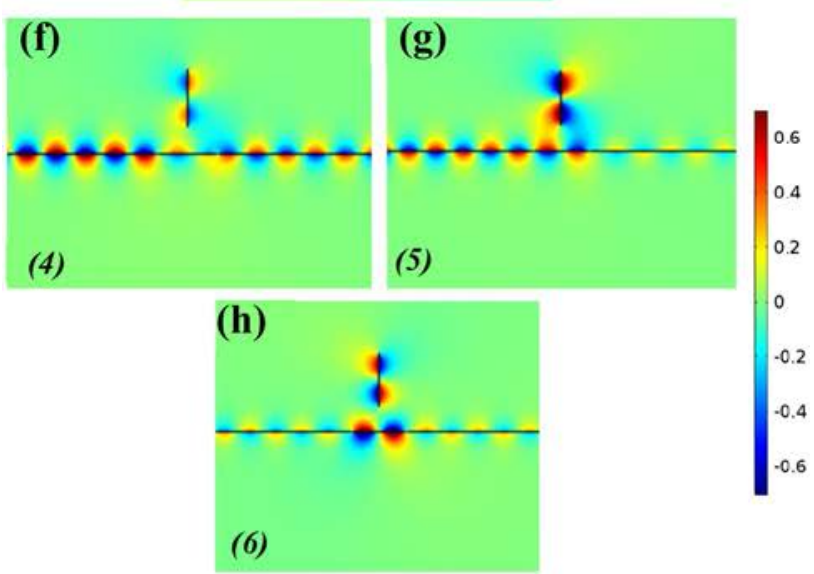

(6)

The parameters of the structure are set as in Fig. 9b. A fairly good fitting can be observed despite some deviations, which we relate (as mentioned earlier) to the approximations of the 

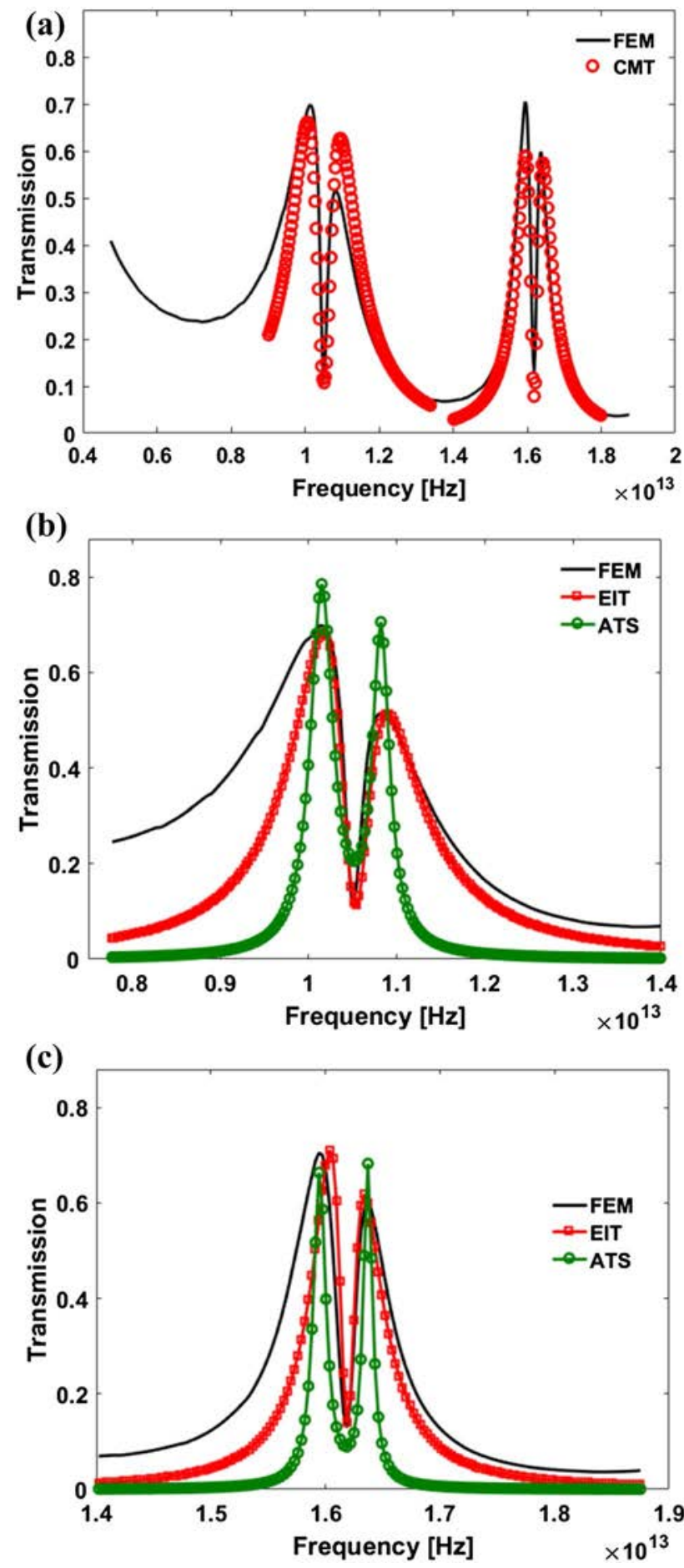

Fig. 10 a Transmission spectra based on the CMT model (red circles) and the FEM method (black solid line). The parameters of the system are fixed as in Fig. 9b. b ATS/EIT-fitting curves of the FEM spectrum at the low frequency resonance. $\mathbf{c}$ Same as in $\mathbf{b}$ but around the high frequency resonance

CMT model and the rough estimations of the fitting parameters $\left(Q_{\mathrm{ov}}, Q_{\mathrm{oh}}, Q_{\mathrm{e}}\right.$ and $\left.Q_{\mathrm{c}}\right)$.

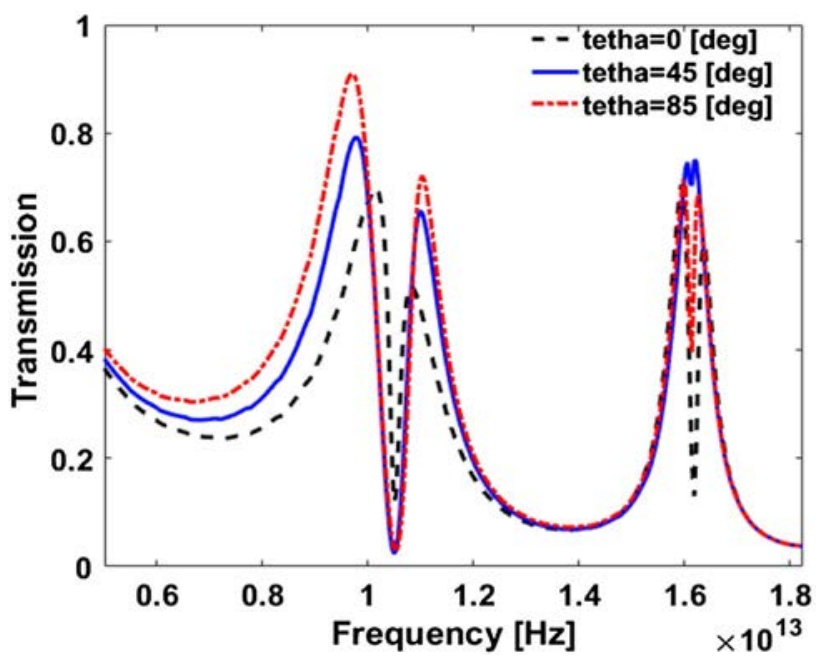

Fig. 11 a Transmission spectrum for three different values of $\theta=0^{\circ}$, $45^{\circ}$ and $85^{\circ}$, with $d=120 \mathrm{~nm}, l_{1}=l_{2}=250 \mathrm{~nm}, g=10 \mathrm{nmand}$ $E_{\mathrm{F} 1}=160 \mathrm{meV}$

To get a quantitative answer as to what is the origin of the PIA-like dips (EIT or ATS), we set out to perform the ATS/EIT fitting of the simulation results. Specifically, we used the equations provided in [32-34] for the ATS-fitting function as follows:

$T_{\mathrm{ATS}}=\frac{C_{1}}{\left(\omega-\delta_{1}\right)^{2}+\Gamma_{1}^{2}}+\frac{C_{2}}{\left(\omega-\delta_{2}\right)^{2}+\Gamma_{2}^{2}}$.

Whereas for the EIT-fitting function, we have

$T_{\mathrm{EIT}}=\frac{C_{1}}{\left(\omega-\omega_{\mathrm{R}}\right)^{2}+\Gamma_{1}^{2}}-\frac{C_{2}}{\left(\omega-\omega_{\mathrm{R}}\right)^{2}+\Gamma_{2}^{2}}$,

where $C_{\mathrm{k}}, \delta_{\mathrm{k}}$ and $\Gamma_{\mathrm{k}}$ are the free fitting parameters. The corresponding results are depicted in Fig. 10b, c around the first and second order resonances, respectively. The EIT function truly provides the best fitting to the numerically based spectrum and does capture all its features; this is even more visible at around the high frequency resonance. As a result, the observed resonances can be mainly attributed to the EIT phenomenon rather than ATS.

Given that EIT is the principal cause behind the induced dips, it is worth adding that the destructive interferences accompanying EIT and taking place here between the two ribbons can be explained in analogy with a Mach-Zehnder interferometer as in [43]. As a matter of fact, the incident light takes two path ways through the two ribbons: the direct path through the horizontal ribbon and the indirect path through the vertical ribbon and the resulting two waves interfere destructively at the exit of the system giving rise to the observed PIA-transmission dips. 
To investigate the possibility of improving the quality factor of the two PIA resonances, we have studied the evolution of the transmission spectrum of the system as a function of the upper ribbon orientation with respect to $y$-axis, that is, the angle $\theta$. In Fig. 11, we have plotted the transmission coefficient evolution of the structure for three values of $\theta=0^{\circ}, 45^{\circ}$ and $85^{\circ}$ (the remaining parameters being kept as before). One observes that the first order PIA resonance at $\theta=0^{\circ}$ is clearly the best case as the FWHM increases as function of $\theta$. This may be related to the fact that when the upper ribbon is tilted with an angle $\theta$, its coupling with the lower ribbon is more effective, leading to the increase of the coupling strength between the ribbons. As a result, the FWHM of the transmission dip is increased.

Nevertheless, intriguingly the behavior of the second order PIA resonance is quite different. Indeed, increasing $\theta$ leads to the improvement of the Q-factor and the height of the transmission dip (or its excitation rate) reaches a minimum around $\theta=45^{\circ}$. Then, the height of the dip becomes higher again. Overall, for this PIA resonance, the Q-factor is best at high values of $\theta$. This behavior may be related to the ribbon second order mode structure/symmetry which is quite different from the first order mode. The parameter $d$ representing the coupling intensity between the dark mode and the bright mode also has an impact on the Q-factor. Indeed, the evolution of the transmission spectrum of the system with $d$ (not shown here) indicates that the latter should be taken as big as possible, to get higher Q-values. Nonetheless, bigger $d$ results in less deep transmission dips which means that a trade-off should be reached at this point.

PIA resonances gives rise to fast light features [29]. To show this, we present in Fig. 12 the delay time evolution

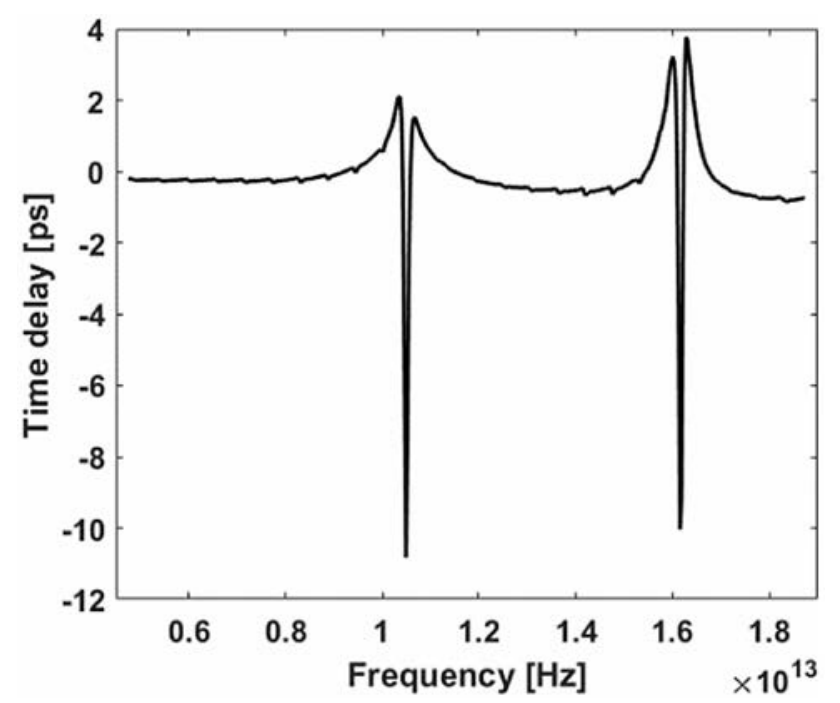

Fig. 12 Delay time of the structure in Fig. $1 \mathrm{~b}$ as a function of the frequency for $d=120 \mathrm{~nm}$ and $\theta=0^{\circ}$, with $l_{1}=l_{2}=250 \mathrm{~nm}$, $g=10 \mathrm{~nm}$ and $E_{\mathrm{F} 1}=160 \mathrm{meV}$

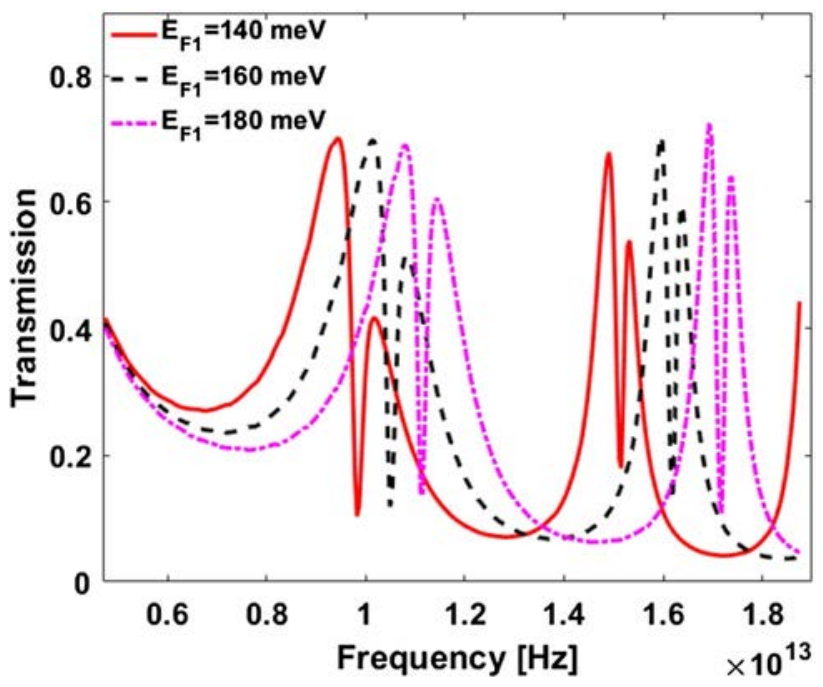

Fig. 13 Transmission spectra of the system for different values of $E_{\mathrm{F} 1}$. Remaining parameters are the same as in Fig. $8 \mathrm{~b}$

versus the frequency for $d=120 \mathrm{~nm}, \theta=0^{\circ}$. It can be observed that the delay time becomes negative at the two PIA windows, indicating a time advance.

Therefore, this character leads to unique fast light feature in the proposed graphene-based device. Let us mention that fast and slow light exhibit potential applications in various fields: telecommunication, interferometry and optical memories [44].

To show the possibility to tune the PIA resonances, we have plotted in Fig. 13 the transmission spectra of the system for different values of the Fermi level $E_{F 1}$, while keeping the remaining parameters fixed. One notices the blue shift of the PIA dips with increasing the Fermi energy level. We justify this behavior as previously based on the simple Eq. (9) relating the resonance wavelength of a GNR with its Fermi energy level.

\section{Conclusion}

In this paper, we have studied numerically and theoretically the possibility of realizing plasmon-induced-like transparency (PIT-like) and plasmon-induced absorption (PIA) resonances in a novel ultra-compact graphene-based-structure. The geometry design of the structure consists in a GBW side-coupled to two GNRs placed either in inverted L-or T-shapes. Both forms of the ribbons give rise to lambdalike state in analogy with the three atomic-level systems. The whole structure is embedded in a $\mathrm{SiO}_{2}$ dielectric material to suit practical applications. When the ribbons are in an inverted L-shape configuration, we have shown that two PIT-like resonances may be obtained by the system. Temporal coupled mode theory (CMT) is provided to fit 
the FEM-based numerical results. The physical mechanism of the PIT-like peaks is unveiled as being caused by mode splitting phenomenon, the so-called Autler-Townes splitting rather than EIT effect. This has been demonstrated by performing the EIT/ATS fitting of the numerical results. In particular, we have shown that increasing the coupling distance strength between the two GNR resonators, results in a cross-over regime where both EIT/ATS contribute to the PIT-like peaks. The improvement of the Q-factor of the resonances is investigated. In addiion, the slow-light effect within the double transparency windows is shown. On the other hand, PIA transmission dips are generated by the system when the two GNRs are set in an inverted T-shape. Here also the two ribbons act like dark and bright modes, respectively. Fitting of the FEM-based results is carried-out using the CMT model. By performing the EIT/ATS fitting of the simulated transmission spectrum, we have shown that the induced dips originate from the EIT effect rather than ATS. Hence, destructive interferences take place between the GNRs at the dip frequencies, leading to the formation of the PIA windows. The amelioration of the Q-factor of the PIA resonances as well as the associated fast-light features are showcased. These results may have potential applications in the realization of graphene-based THz-integrated optical devices such as filters, sensors and ultra-fast optical switching.

\section{References}

1. X. Zhang, N. Xu, K. Qu, Z. Tian, R. Singh, J. Han et al., Electromagnetically induced absorption in a three-resonator metasurface system. Sci. Rep. 5, 10737 (2015)

2. S. Zhang, D.A. Genov, Y. Wang, M. Liu, X. Zhang, Plasmoninduced transparency in metamaterials. Phys. Rev. Lett. 101, 047401 (2008)

3. M. Fleischhauer, A. Imamoglu, J.P. Marangos, Electromagnetically induced transparency: optics in coherent media. Rev. Mod. Phys. 77, 633 (2005)

4. K. Okamoto, D. Tanaka, R. Degawa, X. Li, P. Wang, S. Ryuzaki et al., Electromagnetically induced transparency of a plasmonic metamaterial light absorber based on multilayered metallic nanoparticle sheets. Sci. Rep. 6, 36165 (2016)

5. C. Zhao, S. Xiaokang, J. Rongzhen, D. Gaoyan, W. Lulu, L. Yu et al., Tunable electromagnetically induced transparency in plasmonic system and its application in nanosensor and spectral splitting. IEEE Photonics J. 7, 4801408 (2015)

6. H. Lu, X. Liu, D. Mao, Plasmonic analog of electromagnetically induced transparency in multinanoresonator-coupled waveguide systems. Phys. Rev. A 85, 053803 (2012)

7. J. Guo, Plasmon-induced transparency in metal-insulator-metal waveguide side-coupled with multiple cavities. Appl. Opt. 53, 1604 (2014)

8. H.J. Li, L.L. Wang, B.H. Zhang, X. Zhai, Tunable edge-modebased mid-infrared plasmonically induced transparency in the coupling system of coplanar graphene ribbons. Appl. Phys. Express 9, 012001 (2015)
9. J. Wang, X. Liang, S. Liu, Tunable multimode plasmon-induced transparency with graphene side-coupled resonators. J. Appl. Phys. 55, 022201 (2016)

10. G. Cao et al., Sensing analysis based on plasmon induced transparency in nanocavity coupled waveguide. Opt. Express 23, 20313 (2015)

11. J. Wang et al., A novel planar metamaterial design for electromagnetically induced transparency and slow light. Opt. Express 21, 25159 (2013)

12. S.A. Mikhailov, K. Ziegler, New electromagnetic mode in graphene. Phys. Rev. Lett. 99, 016803 (2007)

13. W. Gao, J. Shu, C. Qiu, Q. Xu, Excitation of plasmonic waves in graphene by guided-mode resonances. ACS Nano. 6, 7806 (2012)

14. K. Geim, K.S. Novoselov, The rise of graphene. Nat. Matter. 6, 183 (2007)

15. Z. Su, X. Chen, J. Yin, X. Zhao, Graphene-based terahertz metasurface with tunable spectrum splitting. Opt. Lett. 41, 3799 (2016)

16. L. Luo, K. Wang, K. Guo, F. Shen, X. Zhang, Z. Yin, Z. Guo, Tunable manipulation of terahertz wavefront based on graphene metasurfaces. J. Opt. 19, 115104 (2017)

17. Z. Fang, Z. Liu, Y. Wang, P.M. Ajayan, P. Nordlander, N.J. Halas, Graphene-antenna sandwich photodetector. Nano Lett. 12, 3808 (2012)

18. Z. Fang, Y. Wang, A.E. Schlather, Z. Liu, P.M. Ajayan, F.J. García de Abajo, P. Nordlander, X. Zhu, N.J. Halas, Active tunable absorption enhancement with graphene nanodisk arrays. Nano Lett. 14, 299 (2013)

19. Y. Nikitin, F. Guinea, F.J. Garcia-Vidal, L. Martin-Moreno, Edge and waveguide terahertz surface plasmon modes in graphene microribbons. Phys. Rev. B 84, 161407 (2011)

20. X. Zhu, W. Yan, N.A. Mortensen, S. Xiao, Bends and splitters in graphene nanoribbon waveguides. Opt. Express 21, 3486 (2013)

21. F. Xing, Z.B. Liu et al., Sensitive real-time monitoring of refractive indexes using a novel graphene-based optical sensor. Sci. Rep. 2, 908 (2012)

22. M. Pan, Z. Liang et al., Tunable angle-independent refractive index sensor based on Fano resonance in integrated metal and graphene nanoribbons. Sci. Rep. 6, 29984 (2016)

23. S.X. Xia, X. Zhai et al., Multi-band perfect plasmonic absorptions using rectangular graphene gratings. Opt. Lett. Lett. 42, 3052 (2017)

24. L. Wang et al., Tunable control of electromagnetically induced transparency analogue in a compact graphene-based waveguide. Opt. Lett. 40, 2325 (2015)

25. S.X. Xia, X. Zhai et al., Plasmonically induced transparency in double-layered graphene nanoribbons. Photon. Res. 6, 692 (2018)

26. A. Lezama et al., Electromagnetically induced absorption. Phys. Rev. A 59, 4732 (1999)

27. J. He et al., Ultra-narrow band perfect absorbers based on plasmonic analog of electromagnetically induced absorption. Opt. Express 23, 6083 (2015)

28. X. Zhang et al., Electromagnetically induced absorption in a threeresonator metasurface system. Sci. Rep. 5, 10737 (2015)

29. M. Wen et al., Dynamically tunable plasmon-induced absorption in resonator-coupled graphene waveguide. Europhys. Lett. 116, 44004 (2017)

30. C. Liu et al., Observation of coherent optical information storage in an atomic medium using halted light pulses. Nature. 409, 490 (2001)

31. F. Xia et al., Ultracompact optical buffers on a silicon chip. Nature Photon. 1, 65 (2007)

32. B. Peng, ŞK. Özdemir et al., what is and what is not electromagnetic induced transparency in whispering-gallery microcavities. Nat. Commun. 5, 5082 (2014) 
33. L. Giner, L. Veissier et al., Experimental investigation of the transition between Autler-Townes splitting and electromagnetically induced transparency models. Phys. Rev. A 87, 013823 (2013)

34. J. Liu, H. Yang et al., Experimental distinction of Autler-Townes splitting from electromagnetically induced transparency using coupled mechanical oscillators system. Sci. Rep. 6, 19040 (2016)

35. J. Chen et al., Optical nano-imaging of gate-tunable graphene plasmons. Nature. 487, 77 (2012)

36. Y. Francescato et al., Strongly confined gap plasmon modes in graphene sandwiches and graphene-on-silicon. New J. Phys. 15, 063020 (2013)

37. S.X. Xia et al., Dynamically tunable plasmonically induced transparency in sinusoidally curved and planar graphene layers. Opt. Express 24, 17886 (2016)

38. Q. Lin, X. Zhai et al., Combined theoretical analysis for plasmoninduced transparency in integrated graphene waveguides with direct and indirect couplings. EPL. 111, 34004 (2015)

39. H. Lu, X. Liu et al., Plasmonic analog of electromagnetically induced transparency in multi-nanoresonator-coupled waveguide systems. Phys. Rev. A 85, 053803 (2012)
40. M.L. Ladron de Guevara, F. Claro et al., Ghost Fano resonance in a double quantum dot molecule attached to leads. Phys. Rev. B 67, 195335 (2003)

41. C. Hu, L. Wang et al., Tunable double transparency windows induced by single subradiant element in coupled graphene plasmonic nanostructure. Appl. Phys. Express 9, 052001 (2016)

42. T. Zhang, J. Zhou, Plasmon induced absorption in a graphenebased nanoribbon waveguide system and its applications in logic gate and sensor. J. Phys. D: Appl. Phys. 51, 055103 (2018)

43. E.H. El Boudouti et al, Experimental and theoretical evidence for the existence of photonic bandgaps and selective transmissions in serial loop structures. J. Appl. Phys. 95.1102 (2004)

44. W. Boyd, Slow and fast light: fundamentals and applications. J. Mod. Opt. 56, 1908 (2009)

Publisher's Note Springer Nature remains neutral with regard to jurisdictional claims in published maps and institutional affiliations. 